\title{
Atualizações sobre o tratamento do Hiperparatireoidismo Secundário: uma revisão de literatura nos últimos 5 anos
}

\author{
Updates on the treatment of Secondary Hyperparathyroidism: a literature review in the last 5 years \\ Actualizaciones sobre el tratamiento del Hiperparatiroidismo Secundario: una revisión de la
}

literatura en los últimos 5 años

Recebido: 19/11/2021 | Revisado: 26/11/2021 | Aceito: 20/12/2021 | Publicado: 03/01/2022

\author{
Alice Barroso Guimarães \\ ORCID: https://orcid.org/0000-0002-2033-7992 \\ Universidade Federal do Pará, Brasil \\ E-mail: alice.guimaraes@ics.ufpa.br \\ Fernanda Protázio Silva \\ ORCID: https://orcid.org/0000-0003-0696-8138 \\ Universidade Federal do Pará, Brasil \\ E-mail: fernanda.protazios13@gmail.com \\ João Marcos Rodrigues Silva \\ ORCID: https://orcid.org/0000-0001-8169-0290 \\ Universidade Federal do Pará, Brasil \\ E-mail: rodriguessilvajoaomarcos@gmail.com \\ Ana Carolina Sardo de Oliveira Pinheiro \\ ORCID: https://orcid.org/0000-0002-2405-8114 \\ Universidade Federal do Pará, Brasil \\ E-mail: sardcarolina@gmail.com \\ João Victor Machado Cristo \\ ORCID: https://orcid.org/0000-0001-7784-8892 \\ Universidade Federal do Pará, Brasil \\ E-mail: victorcristo2019@gmail.com \\ Filipe Santos da Silva \\ ORCID: https://orcid.org/0000-0003-3461-5698 \\ Universidade Federal do Pará, Brasil \\ E-mail: filipe.silva@ics.ufpa.br \\ Letícia Takanashi Baseggio \\ ORCID: https://orcid.org/0000-0003-3077-4704 \\ Universidade Federal do Pará, Brasil \\ E-mail: leticia_takanashi@hotmail.com \\ Fernanda de Souza Parente \\ ORCID: https://orcid.org/0000-0003-3831-6011 \\ Universidade Federal do Pará, Brasil \\ E-mail: feesp7@gmail.com \\ Bruno Souza dos Santos \\ ORCID: https://orcid.org/0000-0002-5174-7783 \\ Centro Universitário do Estado Pará, Brasil \\ E-mail: brunosantosmfc@gmail.com
}

\begin{abstract}
Resumo
Introdução: A elevação persistente nas concentrações plasmáticas do paratormônio (PTH) é responsável por um problema conhecido como hiperparatireoidismo, classificado a partir da origem da quebra da fisiologia normal. No hiperparatireoidismo secundário (HPTS), há alguma disfunção metabólica que leva ao estímulo das glândulas paratireoides, como a doença renal crônica (DRC), causa mais comum. A paratireoidectomia (PTX) possui algumas indicações no tratamento do HPTS, entretanto, condutas como adequação da dieta, reposição de vitamina D ou uso de drogas que provoquem o controle da secreção do PTH geram mais benefícios aos pacientes em detrimento da intervenção cirúrgica. Objetivo: Realizar uma revisão de literatura em base de dados sobre as terapias disponíveis para pacientes que apresentam hiperparatireoidismo secundário. Metodologia: Realizou-se um levantamento de artigos em um período de 5 anos no banco de dados do Pubmed sobre as terapias disponíveis para pacientes que apresentam HPTS, localizando-se, ao final, 19 artigos. Resultados e Discussão: A partir dessa análise, observou-se que novos cálcio-miméticos como Etelcalcetide e Evocalcet demonstraram-se uma alternativa ao Cinalcacet, uma vez que possuem níveis similares de eficácia e segurança, mas com vantagens em relação ao uso e adesão. Além
\end{abstract}


desses, Colecalciferol e Paricalcitol (análogos do calcitriol) e DP0001 (novo ativador do receptor de vitamina D) mostraram-se eficientes na redução do PTH sérico. Conclusão: Apesar de apontar alternativas promissoras, todos os estudos utilizados apresentaram limitações. Portanto, outros estudos devem ser desenvolvidos, com a finalidade de definir recursos farmacológicos e técnicos capazes de se sobreporem aos tratamentos adotados atualmente.

Palavras-chave: Hiperparatireoidismo Secundário; Terapêutica; Tratamento Farmacológico; Paratireoidectomia.

\begin{abstract}
Introduction: Persistent elevation in plasmatic parathyroid hormone (PTH) concentrations is responsible for a problem known as hyperparathyroidism, classified according to the origin of the breakdown of normal physiology. At the secondary hyperparathyroidism (SHPT), there is some metabolic dysfunction that leads to stimulation of the parathyroid glands, such as chronic kidney disease (CKD), the most common cause. Parathyroidectomy (PTX) has some indications in the treatment of SHPT, however, approaches such as diet adequacy, vitamin D replacement or use of drugs that control PTH secretion generate more benefits to patients than surgical intervention. Objective: To conduct a database literature review on available therapies for patients with secondary hyperparathyroidism. Methodology: A survey of articles was carried out over a period of 5 years in the Pubmed database on available therapies for patients with SHPT, finding, in the end, 19 articles. Results and Discussion: From this analysis, it was observed that new calcium-mimetics such as Etelcalcetide and Evocalcet proved to be an alternative to Cinalcacet, as they have similar efficacy and safety, but with advantages regarding use and adherence. In addition to these, Colecalciferol and Paricalcitol (calcitriol analogues) and DP0001 (new vitamin D receptor activator) are efficient in reducing serum PTH. Conclusion: Despite pointing out promising alternatives, all studies had limitations. Therefore, other studies must be developed, with a goal of defining pharmacological and technical resources capable of superimposing the treatments currently adopted.
\end{abstract}

Keywords: Secondary Hyperparathyroidism; Therapy; Pharmacological Treatment; Parathyroidectomy.

\title{
Resumen
}

Introducción: La elevación persistente de las concentraciones plasmáticas de la hormona paratiroidea (PTH) es responsable de un problema conocido como hiperparatiroidismo, que se clasifica de acuerdo con el origen de la ruptura de la fisiología normal. En el hiperparatiroidismo secundario (HPTS), existe alguna disfunción metabólica que conduce a la estimulación de las glándulas paratiroides, como la enfermedad renal crónica (ERC), la causa más común. La paratiroidectomía (PTX) tiene algunas indicaciones en el tratamiento de HPTS, sin embargo, enfoques como la adecuación de la dieta, el reemplazo de vitamina D o el uso de fármacos que controlan la secreción de PTH generan más beneficios para los pacientes que la intervención quirúrgica. Objetivo: Realizar una revisión de la literatura de la base de datos sobre las terapias disponibles para pacientes con hiperparatiroidismo secundario. Metodología: Se realizó un relevamiento de artículos durante un período de 5 años en la base de datos Pubmed sobre terapias disponibles para pacientes con HPTS, encontrándose, al final, 19 artículos. Resultados y Discusión: A partir de este análisis, se observó que nuevos miméticos de calcio como Etelcalcetide y Evocalcet demostraron ser una alternativa al Cinalcacet, ya que tienen similar eficacia y seguridad, pero con ventajas en cuanto a uso y adherencia. Además de estos, el colecalciferol y el paricalcitol (análogos del calcitriol) y el DP0001 (nuevo activador del receptor de vitamina D) son eficaces para reducir la PTH sérica. Conclusión: A pesar de señalar alternativas prometedoras, todos los estudios tenían limitaciones. Por tanto, es necesario desarrollar otros estudios, con la intención de definir recursos farmacológicos y técnicos capaces de reemplazar los tratamientos adoptados actualmente.

Palabras clave: Hiperparatiroidismo secundario; Terapia; Tratamiento farmacológico; Paratiroidectomía.

\section{Introdução}

No Brasil, o número total estimado de pacientes em diálise passou de 77.589, em 2009, para 133.464, em 2018. Isso representa um aumento de mais de 72\%, em 10 anos, na quantidade de pacientes com DRC que precisam de diálise (Neves, 2020). Atrelado a esse fato, dentre as complicações mais frequentes que acometem esses pacientes, o HPTS atinge aproximadamente 44\% deles (Custódio, 2013). Além disso, o HPTS é considerado uma importante causa de mortalidade e de perda da qualidade de vida em pacientes com DRC (Porto, 2016).

A elevação persistente nas concentrações plasmáticas do paratormônio (PTH) é responsável por um problema conhecido como hiperparatireoidismo (Golan, 2014, pg. 522). Essa condição é classicamente categorizada em hiperparatireoidismo primário (HPTP) e secundário (HPTS). A base dessa classificação é a origem da quebra da fisiologia normal da glândula paratireoide: no HPTP, há uma doença da própria paratireoide que leva à hipersecreção glandular (adenomas ou hiperplasia, por exemplo); e já no HPTS, a hipersecreção hormonal ocorre devido a alguma disfunção 
metabólica que leva ao estímulo das glândulas paratireoides, como a DRC, por exemplo, que é a causa mais comum (Xavier, 2020).

O PTH atua sobre os osteoblastos, osteócitos e osteoclastos dos ossos. No rim, seus efeitos são direcionados para aumentar a reabsorção tubular de cálcio e aumentar a excreção de fosfato (Bilezikian, 2018). A elevação do PTH produz alta atividade de osteoblastos e osteoclastos nos ossos, o que eleva a concentração do cálcio iônico extracelular, ao passo em que tende a deprimir a concentração dos íons fosfato, pelo aumento da excreção renal. Com o tempo, tal desequilíbrio no organismo afetado pode gerar doença óssea, formação de cálculos renais e hipercalcemia. Nessa última, os efeitos dos níveis elevados de cálcio incluem a depressão do sistema nervoso central e periférico, fraqueza muscular, constipação, dor abdominal, úlcera péptica, falta de apetite e os problemas cardíacos (Guyton, 2013, pg. 1018 -1019).

Nos casos de HPTS, o procedimento cirúrgico é indicado em situações de hipercalcemia persistente, calcificação ectópica, lesão óssea grave e prurido intratável (Bringhust, 1998), entretanto, existem condições em que o tratamento clínico pode ser mais adequado. Condutas que passam pela adequação da dieta, reposição de vitamina $\mathrm{D}$ e por drogas que provoquem o controle da secreção do PTH intacto (iPTH) como cálcio-miméticos, ativadores seletivos dos receptores de vitamina D (paricalcitol), calcitriol e análogos da vitamina D, são todas opções farmacológicas e não farmacológicas para o tratamento dessa doença que geram mais benefícios aos pacientes em detrimento da intervenção cirúrgica (Custódio, 2013).

Nesse sentido, o presente trabalho teve como objetivo realizar uma revisão de literatura em base de dados sobre as terapias disponíveis para pacientes que apresentam hiperparatireoidismo secundário.

\section{Metodologia}

Foi realizado, no mês de fevereiro de 2021, um levantamento na literatura para confecção de revisão do tipo narrativa em um período de 5 anos (2016 a 2021) no banco de dados do Pubmed. Para a abordagem, utilizaram-se as palavraschaves, organizadas da seguinte maneira: ("hyperparathyroidism secondary" OR "hiperparatireoidismo secundário" OR "SHPT") AND ("parathyroidectomy" OR "paratireoidectomia" OR "treatment" OR "tratamento"). Em seguida, os resultados obtidos foram filtrados de acordo com o período citado, os idiomas pretendidos (português e inglês) e o tipo de estudo, sendo considerados apenas ensaios clínicos e ensaios clínicos randomizados. Ademais, só foram adicionados os artigos que apresentavam a temática citada em seu abstract e que estivessem gratuitamente disponíveis para acesso. Nesse sentido, foram encontrados, na data da pesquisa, 20 artigos. Após leitura dos resumos e textos completos, e definição do tema a ser abordado no trabalho, foram selecionados 19 artigos para serem utilizados na revisão de literatura. Foram excluídos artigos que não apresentavam foco para o tema delimitado.

\section{Resultados e Discussão}

\begin{tabular}{|c|c|c|c|c|c|}
\hline Título & Tipo de estudo & Ano & Autor & Tipo de tratamento & \multicolumn{1}{c|}{ Resultados principais } \\
\hline $\begin{array}{c}\text { The effectiveness of } \\
\text { cinacalcet: a randomized, } \\
\text { open label study in } \\
\text { chronic hemodialysis } \\
\text { patients with severe } \\
\text { secondary } \\
\text { hyperparathyroidism. }\end{array}$ & $\begin{array}{c}\text { Estudo } \\
\text { observacional } \\
\text { analítico }\end{array}$ & 2019 & $\begin{array}{c}\text { Susantitaphong et } \\
\text { al. }\end{array}$ & Cinacalcet & $\begin{array}{l}\text { - O estudo demonstrou a redução efetiva do iPTH } \\
\text { sérico e tendência à redução no tamanho da } \\
\text { glândula em pacientes com nível basal por volta de } \\
800-1600 \text { pg/mL. } \\
\text { - Os achados reforçam que pacientes em } \\
\text { hemodiálise crônica com níveis }>1600 \text { devem ser } \\
\text { aconselhados à cirurgia. } \\
\text { - O cinacalcet pode reduzir a fosfatina FGF-23 em } \\
\text { casos de hemodiálise crônica com níveis médios de } \\
\text { iPTH por volta de 1400 pg/mL. No entanto, o } \\
\text { benefício a longo-prazo deste achado ainda } \\
\text { necessita de mais estudos. }\end{array}$ \\
\hline
\end{tabular}




\begin{tabular}{|c|c|c|c|c|c|}
\hline & & & & & $\begin{array}{l}\text { - O cinacalcete pôde estabilizar a calcificação } \\
\text { vascular no estudo, e apenas } 15 \% \text { dos pacientes } \\
\text { tiveram progressão desta. } \\
\text { - Apesar da paratireoidectomia reduzir de maneira } \\
\text { eficaz os níveis de PTH na maioria dos pacientes, a } \\
\text { melhora no controle bioquímico no pós-cirúrgico } \\
\text { pode não ser perfeita. }\end{array}$ \\
\hline $\begin{array}{l}\text { PTH-dependence of the } \\
\text { effectiveness of } \\
\text { cinacalcet in } \\
\text { hemodialysis patients } \\
\text { with secondary } \\
\text { hyperparathyroidism }\end{array}$ & $\begin{array}{c}\text { Estudo } \\
\text { observacional } \\
\text { analítico }\end{array}$ & 2016 & Akizawa et al. & Cinacalcet & $\begin{array}{l}\text { - A iniciação com cinacalcet foi mais eficaz em } \\
\text { pacientes que tinham níveis mais elevados de iPTH } \\
\text { no início do estudo, em dois desfechos clínicos } \\
\text { importantes: morte devido a qualquer causa e um } \\
\text { composto de hospitalização cardiovascular e } \\
\text { mortalidade. } \\
\text { - Esses achados podem auxiliar as decisões de } \\
\text { médicos e pacientes em diálise a considerarem o } \\
\text { uso do cinacalcet, dada a gravidade do HPTS do } \\
\text { paciente. }\end{array}$ \\
\hline $\begin{array}{l}\text { A Randomized Study } \\
\text { Comparing } \\
\text { Parathyroidectomy with } \\
\text { Cinacalcet for Treating } \\
\text { Hypercalcemia in Kidney } \\
\text { Allograft Recipients with } \\
\text { Hyperparathyroidism. }\end{array}$ & $\begin{array}{c}\text { Estudo } \\
\text { observacional } \\
\text { analítico }\end{array}$ & 2016 & Cruzado et al. & $\begin{array}{c}\text { Paratireoidectomia } \\
\text { Cinacalcet }\end{array}$ & $\begin{array}{l}\text { - O estudo mostrou que a paratireoidectomia (PTX) } \\
\text { subtotal é superior ao cinacalcet para corrigir } \\
\text { hipercalcemia em pacientes transplantados renais } \\
\text { com HPT persistente. } \\
\text { - Todos os pacientes submetidos à cirurgia e } \leq 70 \% \\
\text { em uso de cinacalcet atingiram normocalcemia. } \\
\text { - PTX subtotal foi associada com melhora da } \\
\text { densidade óssea mineral, regulação dos } \\
\text { biomarcadores de volume ósseo e maior redução do } \\
\text { iPTH. } \\
\text { - Ambos os tratamentos foram associados a não } \\
\text { progressão da calcificação vascular. } \\
\text { - Redução da taxa de filtração glomerular foi } \\
\text { observada em ambos os grupos, embora mais } \\
\text { acentuada com o cinacalcet. } \\
\text { - Os efeitos adversos gastrointestinais observados } \\
\text { no tratamento com o fármaco podem ser associados } \\
\text { à hipovolemia e disfunção renal. } \\
\text { - Hospitalização precoce foi de 13\% após PTX e } \\
7 \% \text { com o cinacalcet. A maior complicação após a } \\
\text { cirurgia foi a hipocalcemia. }\end{array}$ \\
\hline $\begin{array}{l}\text { An integrated analysis of } \\
\text { safety and tolerability of } \\
\text { etelcalcetide in patients } \\
\text { receiving hemodialysis } \\
\text { with secondary } \\
\text { hyperparathyroidism. }\end{array}$ & $\begin{array}{c}\text { Estudo } \\
\text { observacional } \\
\text { analítico }\end{array}$ & 2019 & Block et al. & Etelcalcetide & $\begin{array}{l}\text { - O perfil de segurança do etelcalcitide no estudo } \\
\text { não revelou sinais além dos já reportados. As } \\
\text { reações adversas foram majoritariamente ligadas à } \\
\text { diminuição do cálcio sérico (QT prolongado, } \\
\text { parestesia, espasmos musculares e mialgia) ou } \\
\text { sintomas gastrointestinais; os demais foram } \\
\text { hipercalemia, insuficiência cardíaca, } \\
\text { hipofosfatemia e cefaleia. } \\
\text { - Não foram encontradas diferenças nas taxas de } \\
\text { hipocalcemia entre pacientes tratados com } \\
\text { etelcalcetide ou cinacalcet. } \\
\text { - Taxas de fratura no grupo com etelcalcetide } \\
\text { foram quase metade das observadas no grupo } \\
\text { placebo, e não houve aumento com exposição } \\
\text { prolongada. } \\
\text { - É possível que a redução no nível de cálcio possa } \\
\text { desencadear instabilidade hemodinâmica e que a } \\
\text { hipocalcemia rápida ou prolongada pode } \\
\text { potencialmente reduzir a contratilidade do } \\
\text { miocárdio, principalmente em pacientes com } \\
\text { função cardíaca deficiente. Não há evidência de } \\
\text { efeito direto na repolarização cardíaca. }\end{array}$ \\
\hline
\end{tabular}




\begin{tabular}{|c|c|c|c|c|c|}
\hline & & & & & $\begin{array}{l}\text { - Há risco baixo ou nulo de interações com outras } \\
\text { drogas, porém interações farmacodinâmicas podem } \\
\text { ocorrer com fármacos que reduzem o cálcio sérico. }\end{array}$ \\
\hline $\begin{array}{l}\text { Effect of Etelcalcetide vs } \\
\text { Placebo on Serum } \\
\text { Parathyroid Hormone in } \\
\text { Patients Receiving } \\
\text { Hemodialysis With } \\
\text { Secondary } \\
\text { Hyperparathyroidism: } \\
\text { Two Randomized } \\
\text { Clinical Trials. }\end{array}$ & $\begin{array}{l}\text { Estudo } \\
\text { observacional } \\
\text { analítico }\end{array}$ & 2017 & Block et al. & Etelcalcetide & $\begin{array}{l}\text { - O tratamento com etelcalcetide reduziu o cálcio } \\
\text { sérico na maioria dos pacientes, com hipocalcemia } \\
\text { sintomática evidente. } \\
\text { - O efeito redutor de cálcio do fármaco teve menor } \\
\text { nível nas semanas } 10 \text { a } 12 \text {, apesar do aumento do } \\
\text { uso de cálcio oral, análogos ativos de vitamina D e } \\
\text { cálcio dialisado em fração substancial. } \\
\text { - Efeitos adversos ocorreram em } 92 \% \text { dos tratados } \\
\text { com etelcalcetide e em } 80 \% \text { dos placebos. Náusea, } \\
\text { vômitos e diarreias foram mais comumente } \\
\text { encontrados no grupo do fármaco, assim como } \\
\text { sintomas relacionados à hipocalcemia. } \\
\text { - Hipercalemia foi reportada com o dobro de } \\
\text { frequência nos tratados com etelcalcetide, porém a } \\
\text { revisão desses eventos não mostrou fatores de risco } \\
\text { consistentes ou eventos associados. } \\
\text { - É preciso cautela no uso do fármaco em conjunto } \\
\text { com outras drogas que possam prolongar o } \\
\text { intervalo QT. } \\
\text { - Não houve diferenças notáveis nas taxas de } \\
\text { morte, IAM, AVC ou convulsões entre os grupos, e } \\
\text { apenas um pequeno desequilíbrio numérico nas } \\
\text { insuficiências cardíacas que demandaram } \\
\text { hospitalização. }\end{array}$ \\
\hline $\begin{array}{l}\text { A phase } 3 \text {, multicentre, } \\
\text { randomized, double- } \\
\text { blind, placebo-controlled, } \\
\text { parallel-group study to } \\
\text { evaluate the efficacy and } \\
\text { safety of etelcalcetide } \\
\text { (ONO-5163/AMG 416), } \\
\text { a novel intravenous } \\
\text { calcimimetic, for } \\
\text { secondary } \\
\text { hyperparathyroidism in } \\
\text { Japanese haemodialysis } \\
\text { patients }\end{array}$ & $\begin{array}{l}\text { Estudo } \\
\text { observacional } \\
\text { analítico }\end{array}$ & 2017 & Fukagawa et al. & Etelcalcetide & $\begin{array}{l}\text { - A administração 3x/semana de etelcalcetide } \\
\text { durante } 12 \text { semanas reduziu o iPTH sérico após } 85 \\
\text { dias de estudo, com significância estatística em } \\
\text { comparação ao placebo. } \\
\text { - Ocorreram náuseas e vômitos no grupo tratado, } \\
\text { no entanto foram eventos leves e não provocaram a } \\
\text { descontinuação do tratamento, demonstrando que o } \\
\text { etelcalcetide foi bem tolerado em pacientes } \\
\text { japoneses. } \\
\text { - O etelcalcetide intravenoso pode ser facilmente } \\
\text { administrado através do retorno do sangue após a } \\
\text { diálise, permitindo uma redução na carga de } \\
\text { comprimidos para os pacientes e fornecendo uma } \\
\text { nova alternativa para maior adesão dos pacientes ao } \\
\text { tratamento de HPTS. }\end{array}$ \\
\hline $\begin{array}{l}\text { One-year safety and } \\
\text { efficacy of intravenous } \\
\text { etelcalcetide in patients } \\
\text { on hemodialysis with } \\
\text { secondary } \\
\text { hyperparathyroidism }\end{array}$ & $\begin{array}{l}\text { Estudo } \\
\text { observacional } \\
\text { analítico }\end{array}$ & 2019 & Bushinsky et al. & Etelcalcetide & $\begin{array}{l}\text { - No geral, } 89,8 \% \text { dos pacientes tiveram um ou } \\
\text { mais eventos adversos emergentes do tratamento. } \\
\text { Os mais comuns foram diminuição do cálcio no } \\
\text { sangue }(43,3 \%) \text {, e sintomas gastrointestinais: } \\
\text { diarreia }(10,8 \%) \text {, vômitos (10,4\%) e náuseas } \\
(9,6 \%) \text {. } \\
\text { - A hipocalcemia sintomática ocorreu em } 3,7 \% \text { dos } \\
\text { pacientes, com sintomas transitórios leve a } \\
\text { moderados, enquanto efeitos adversos associados a } \\
\text { hipercalcemia, como eventos cardíacos, } \\
\text { neuromusculares e convulsões ocorreram em } \\
\text { menos de } 1 \% \text { dos pacientes. } \\
\text { - Observou-se que o etelcalcetide pode reduzir } \\
\text { clinicamente e manter os níveis séricos de PTH } \\
\text { com tratamento de longo prazo. } \\
\text { - Menos de } 5 \% \text { dos pacientes sofreram eventos que } \\
\text { levaram à descontinuação do tratamento com } \\
\text { etelcalcetide, exibindo um perfil de segurança } \\
\text { razoável com reduções sustentadas de PTH, cálcio }\end{array}$ \\
\hline
\end{tabular}




\begin{tabular}{|c|c|c|c|c|c|}
\hline & & & & & e fósforo. \\
\hline $\begin{array}{l}\text { Long-Term Efficacy and } \\
\text { Safety of Evocalcet in } \\
\text { Japanese Patients with } \\
\text { Secondary } \\
\text { Hyperparathyroidism } \\
\text { Receiving Hemodialysis. }\end{array}$ & $\begin{array}{c}\text { Estudo } \\
\text { observacional } \\
\text { analítico }\end{array}$ & 2019 & Yokoyama et al. & Evocalcet & $\begin{array}{l}\text { - O número de pacientes que atingiram a meta de } \\
\text { iPTH aumentou progressivamente com a } \\
\text { administração contínua de evocalcet durante as } 52 \\
\text { semanas do estudo. A maioria deles, no entanto, } \\
\text { realizou tratamento prévio com cinacalcet, o que } \\
\text { pode ter influenciado os parâmetros iniciais. } \\
\text { - A concetração de iPTH diminuiu ainda mais após } \\
\text { a troca para o evocalcet. } \\
\text { - Embora os níveis de iPTH, cálcio e fósforo } \\
\text { tenham aumentado temporariamente após a troca, } \\
\text { tais flutuações podem ser atribuídas à dosagem pré- } \\
\text { terapêutica de cinacalcet, ou ainda, à dose } \\
\text { inicialmente administrada relativamente baixa de } \\
\text { evocalcet (1 ou } 2 \text { mg). } \\
\text { - O evocalcet foi de maneira geral bem tolerado nas } \\
\text { doses administradas, particularmente no que } \\
\text { concerne aos efeitos adversos gastrointestinais. Os } \\
\text { efeitos adversos mais significativos foram } \\
\text { considerados leves; os graves incluíram } \\
\text { insuficiência cardíaca e cardiomiopatia. } \\
\text { - Não ocorreram eventos significativos atribuídos } \\
\text { ao aumento da dose de evocalcet, permitindo sua } \\
\text { intensificação e melhorando a eficácia da droga. } \\
\text { - O tratamento prévio com cinacalcet não ofereceu } \\
\text { preocupações quanto à segurança dos pacientes, e o } \\
\text { perfil de segurança do evocalcet sustenta sua } \\
\text { administração a longo-prazo. }\end{array}$ \\
\hline $\begin{array}{l}\text { Pharmacokinetics, } \\
\text { Pharmacodynamics, and } \\
\text { Safety of the Novel } \\
\text { Calcimimetic Agent } \\
\text { Evocalcet in Healthy } \\
\text { Japanese Subjects: First- } \\
\text { in-Human Phase I Study }\end{array}$ & $\begin{array}{c}\text { Estudo } \\
\text { observacional } \\
\text { analítico }\end{array}$ & 2018 & Akizawa et al. & Evocalcet & $\begin{array}{l}\text { - Após a administração de evocalcet, os níveis de } \\
\text { iPTH e os níveis corrigidos de cálcio diminuíram } \\
\text { proporcionalmente à dose, permanecendo em } \\
\text { níveis baixos constantes em doses crescentes, } \\
\text { demonstrando que o evocalcet teve um efeito } \\
\text { farmacológico semelhante ao do cinacalcet, porém } \\
\text { esse efeito pôde ser alcançado com uma dose mais } \\
\text { baixa comparado ao cinacalcet. } \\
\text { - A modulação CaSR é uma abordagem eficaz para } \\
\text { controlar o HPTS. } \\
\text { - O evocalcet foi seguro e tolerável em doses de até } \\
20 \text { mg no estudo de dose única e até } 12 \text { mg no } \\
\text { estudo de dose múltipla de } 8 \text { dias 1x/dia. Não } \\
\text { ocorreram distúrbios gastrointestinais com a dose } \\
\text { de } 12 \text { mg, levando a crer que o evocalcet pode } \\
\text { causar menos sintomas gastrintestinais do que os } \\
\text { observados para o cinacalcet. }\end{array}$ \\
\hline $\begin{array}{l}\text { Pharmacodynamics of } \\
\text { evocalcet for secondary } \\
\text { hyperparathyroidism in } \\
\text { Japanese hemodialysis } \\
\text { patients }\end{array}$ & $\begin{array}{c}\text { Estudo } \\
\text { observacional } \\
\text { analítico }\end{array}$ & 2019 & Shigematsu et al. & Evocalcet & $\begin{array}{l}\text { - Os eventos adversos ocorreram em } 29,6 \text { e } 62,5 \% \\
\text { dos pacientes que receberam doses múltiplas de } 1 \\
\text { ou } 4 \text { mg, respectivamente, sendo a maioria desses, } \\
\text { leves. } \\
\text { - O evocalcet foi bem tolerado, embora níveis } \\
\text { diminuídos de cálcio no sangue tenham sido } \\
\text { relatados em oito pacientes, resultando na } \\
\text { descontinuação do tratamento } 13 \% \text {. Além disso, os } \\
\text { dados mostraram uma baixa incidência de efeitos } \\
\text { relacionados ao sistema gastrointestinal, mesmo } \\
\text { com a dose máxima de } 12 \text { mg. } \\
\text { - Em comparação com o cinacalcet, o evocalcet } \\
\text { apresenta menos efeitos adversos gastrointestinais, } \\
\text { maior biodisponibilidade, mesmo em baixas doses, } \\
\text { e efeito supressor de iPTH semelhante. } \\
\text { - A respeito de alterações no ECG, foi observada } \\
\text { uma correlação entre o prolongamento do QT }\end{array}$ \\
\hline
\end{tabular}




\begin{tabular}{|c|c|c|c|c|c|}
\hline & & & & & $\begin{array}{l}\text { corrigido de Fridericia (QTcF) e a administração de } \\
\text { evocalcet, devido à diminuição de cálcio no } \\
\text { sangue. }\end{array}$ \\
\hline $\begin{array}{c}\text { Efficacy and safety of } \\
\text { evocalcet in Japanese } \\
\text { peritoneal dialysis } \\
\text { patients. }\end{array}$ & $\begin{array}{l}\text { Estudo } \\
\text { observacional } \\
\text { analítico }\end{array}$ & 2019 & Tsuruya et al. & Evocalcet & $\begin{array}{l}\text { - A administração com ajuste de dose de evocalcet } \\
\text { 1x por dia durante } 32 \text { semanas, seguida por um } \\
\text { tratamento de extensão por } 20 \text { semanas, mostrou } \\
\text { que pacientes com HPTS recebendo diálise } \\
\text { peritoneal atingiram a meta de iPTH. } \\
\text { - Níveis corrigidos de cálcio e fósforo séricos } \\
\text { permaneceram constantes até a } 52^{\mathrm{a}} \text { semana. } \\
\text { - Não foram observadas novas preocupações } \\
\text { quanto à segurança, nem reportadas reações } \\
\text { adversas severas. }\end{array}$ \\
\hline $\begin{array}{l}\text { Phase } 2 \mathrm{~b} \text { study of } \\
\text { evocalcet (KHK7580), a } \\
\text { novel calcimimetic, in } \\
\text { Japanese patients with } \\
\text { secondary } \\
\text { hyperparathyroidism } \\
\text { undergoing } \\
\text { hemodialysis: A } \\
\text { randomized, double- } \\
\text { blind, placebo-controlled, } \\
\text { dose-finding study }\end{array}$ & $\begin{array}{l}\text { Estudo } \\
\text { observacional } \\
\text { analítico }\end{array}$ & 2018 & Akizawa et al. & $\begin{array}{l}\text { Evocalcet } \\
\text { Cinacalcet }\end{array}$ & $\begin{array}{l}\text { - A dependência do evocalcet com a dose utilizada } \\
\text { foi confirmada, bem como a vantagem do evocalcet } \\
\text { em relação ao placebo, começando com uma dose } \\
\text { do fármaco de } 1 \mathrm{mg} \text {. } \\
\text { - O cinacalcet tem potencial para suprimir níveis } \\
\text { séricos de PTH mesmo em HPTS grave. No } \\
\text { entanto, o evocalcet } 2 \text { mg pode ser mais benéfico } \\
\text { para pacientes gravemente afetados em termos de } \\
\text { melhora rápida dos níveis de PTH. } \\
\text { - Estudos comparativos diretos adicionais são } \\
\text { necessários para comparar os fármacos em termos } \\
\text { de sintomas gastrointestinais. }\end{array}$ \\
\hline $\begin{array}{l}\text { Association of Increased } \\
\text { Serum Leptin with } \\
\text { Ameliorated Anemia and } \\
\text { Malnutrition in Stage } 5 \\
\text { Chronic Kidney Disease } \\
\text { Patients after } \\
\text { Parathyroidectomy. }\end{array}$ & $\begin{array}{l}\text { Estudo } \\
\text { observacional } \\
\text { analítico }\end{array}$ & 2016 & Jiang et al. & Leptina sérica & $\begin{array}{l}\text { - Foi demonstrada forte e independente associação } \\
\text { entre baixos níveis séricos de leptina e baixo IMC, } \\
\text { anemia e níveis reduzidos de albumina em } \\
\text { pacientes com doença renal crônica (DRC). } \\
\text { - A normalização dos níveis de PTH possibilitou } \\
\text { melhora na anemia e má nutrição em pacientes com } \\
\text { HPTS severo após a PTX. } \\
\text { - A leptina pode ser promissora para gerar } \\
\text { desfechos favoráveis em pacientes DRC com } \\
\text { dislipidemia. }\end{array}$ \\
\hline $\begin{array}{l}\text { Efficacy and safety of } \\
\text { paricalcitol in children } \\
\text { with stages } 3 \text { to } 5 \text { chronic } \\
\text { kidney disease }\end{array}$ & $\begin{array}{l}\text { Estudo } \\
\text { observacional } \\
\text { analítico }\end{array}$ & 2017 & Webb et al. & Paricalcitol & $\begin{array}{l}\text { - Os dados desses estudos demonstram que o } \\
\text { tratamento de crianças de } 10 \text { a } 16 \text { anos com DRC } \\
\text { nos estágios } 3 \text { a } 5 \text { com paricalcitol oral foi eficaz } \\
\text { na redução dos níveis de iPTH e foi bem tolerado } \\
\text { por longos períodos de teste. } \\
\text { - Além disso, a administração de cápsulas de } \\
\text { paricalcitol para o tratamento de HPTS nos } \\
\text { pacientes entre } 10 \text { e } 16 \text { anos com DRC em estágio } \\
3 \text { ou } 4 \text { parece ser semelhante à observada em } \\
\text { pacientes adultos. Esses resultados apoiam uma } \\
\text { dose inicial de } 1 \mu \text { de paricalcitol em estudos } \\
\text { futuros em crianças de } 10 \text { a } 16 \text { anos com DRC, } \\
\text { bem como na prática clínica. }\end{array}$ \\
\hline $\begin{array}{l}\text { High doses of } \\
\text { cholecalciferol alleviate } \\
\text { the progression of } \\
\text { hyperparathyroidism in } \\
\text { patients with CKD } \\
\text { Stages 3-4: results of a } \\
\text { 12-week double-blind, } \\
\text { randomized, controlled } \\
\text { study }\end{array}$ & $\begin{array}{l}\text { Estudo } \\
\text { observacional } \\
\text { analítico }\end{array}$ & 2018 & Westerberg et al. & Colecalciferol & $\begin{array}{l}\text { - Demonstra-se que uma alta dose diária de } \\
\text { colecalciferol oral ( } 8.000 \text { UI / dia) ameniza o } \\
\text { aumento adicional no PTH e o desenvolvimento de } \\
\text { HPTS na DRC. } \\
\text { - Todos os indivíduos tratados com colecalciferol } \\
\text { tornam-se } 25 \text { (OH) D suficientes após } 3 \text { meses, e } \\
\text { apesar de um aumento substancial nesse composto } \\
\text { e de } 1,25 \text { (OH) D, não houve casos de } \\
\text { hipercalcemia ou perda acelerada da taxa de } \\
\text { filtração glomerular no grupo de tratamento. } \\
\text { - Além disso, o colecalciferol em altas doses não } \\
\text { induz aumento de fosfato ou FGF23, mas, por }\end{array}$ \\
\hline
\end{tabular}




\begin{tabular}{|c|c|c|c|c|c|}
\hline & & & & & $\begin{array}{l}\text { outro lado, não melhora a fadiga ou a força de } \\
\text { preensão manual. }\end{array}$ \\
\hline $\begin{array}{l}\text { Cholecalciferol } \\
\text { Additively Reduces } \\
\text { Serum Parathyroid } \\
\text { Hormone Levels in } \\
\text { Severe Secondary } \\
\text { Hyperparathyroidism } \\
\text { Treated with Calcitriol } \\
\text { and Cinacalcet among } \\
\text { Hemodialysis Patient }\end{array}$ & $\begin{array}{c}\text { Estudo } \\
\text { observacional } \\
\text { analítico }\end{array}$ & 2018 & Zheng et al. & $\begin{array}{l}\text { Colecalciferol } \\
\text { Calcitriol } \\
\text { Cinacalcet }\end{array}$ & $\begin{array}{l}\text { - Um nível de iPTH significativamente mais baixo } \\
\text { foi observado a partir da } 20^{\mathrm{a}} \text { semana no grupo de } \\
\text { estudo com colecalciferol em comparação com o } \\
\text { grupo de placebo, e o iPTH alvo } \leq 300 \mathrm{pg} / \mathrm{mL} \text { foi } \\
\text { alcançado na } 24^{\mathrm{a}} \text { semana no grupo de estudo. } \\
\\
\text { - Cerca de } 89 \% \text {, atingiu } 25 \text {-(OH) D sérico } \geq 30 \mathrm{ng} \text { / } \\
\text { mL no grupo de estudo ao final da pesquisa. Além } \\
\text { disso, o número de pacientes que tiveram aumento } \\
\text { de } 10 \% \text { na densidade mineral óssea (DMO) do colo } \\
\text { do fêmur (CF) foi maior no grupo de estudo em } \\
\text { comparação com o grupo placebo, porém sem } \\
\text { significância estatística. } \\
\text { - O colecalciferol reduziu aditivamente os níveis } \\
\text { séricos de iPTH, melhorou os níveis de } 25-(\mathrm{OH}) \mathrm{D} \\
3 \text { e melhorou a DMO do CF quando usado junto } \\
\text { com cinacalcet ou calcitriol em pacientes com } \\
\text { HPTS grave em hemodiálise. }\end{array}$ \\
\hline $\begin{array}{l}\text { Effectiveness and safety } \\
\text { of a } 6 \text {-month treatment } \\
\text { with paricalcitol in } \\
\text { patients on hemodialysis } \\
\text { with secondary } \\
\text { hyperparathyroidism }\end{array}$ & $\begin{array}{c}\text { Estudo } \\
\text { observacional } \\
\text { analítico }\end{array}$ & 2016 & Olaizola et al. & Paricalcitol & $\begin{array}{l}\text { - Entre os } 26 \text { pacientes registrados, } 19 \text { completaram } \\
\text { os } 6 \text { meses de tratamento. Todos os pacientes } \\
\text { mostraram níveis de referência iPTH reduzidos } \\
\text { (redução média, } 371,8 \mathrm{pg} / \mathrm{mL}) ; 17 \text { pacientes } \\
(89,5 \%) \text { tiveram reduções superiores a } 30 \% \text {. } \\
\\
\text { - O nível sérico médio de cálcio foi aceitável e } \\
\text { nenhum dos pacientes apresentou sintomas clínicos } \\
\text { de hipercalcemia. Os efeitos adversos observados } \\
\text { foram: óbito, dispneia de origem cardíaca, } \\
\text { pneumonia, arritmia, hiperfosfatemia, } \\
\text { hipercalcemia, elevações de TGO e TGP e náuseas, } \\
\text { sendo o mais comumente relatado a } \\
\text { hiperfosfatemia. } \\
\text { - Os resultados deste estudo apoiam o uso de } \\
\text { paricalcitol endovenoso como tratamento eficaz e } \\
\text { bem tolerado para o controle dos níveis de iPTH } \\
\text { em pacientes em hemodiálise com HPTS com } \\
\text { impacto mínimo sobre os teores de cálcio e fósforo. }\end{array}$ \\
\hline $\begin{array}{l}\text { Efficacy of microwave } \\
\text { ablation for severe } \\
\text { secondary } \\
\text { hyperparathyroidism in } \\
\text { subjects undergoing } \\
\text { hemodialysis }\end{array}$ & $\begin{array}{c}\text { Estudo } \\
\text { observacional } \\
\text { analítico }\end{array}$ & 2017 & Diao et al. & $\begin{array}{l}\text { Ablação por micro- } \\
\text { ondas }\end{array}$ & $\begin{array}{l}\text { - Entre os } 26 \text { pacientes submetidos ao tratamento } \\
\text { de ablação por micro-ondas, apenas } 10 \\
\text { responderam, indicando baixa eficácia. } \\
\text { - A complicação mais comum foi a hipocalcemia } \\
(50 \%) \text {. Entre os que responderam ao tratamento, a } \\
\text { concentração de cálcio sérico retornou à } \\
\text { normalidade em } 7 \text { meses. Não houve alterações nas } \\
\text { concentrações de fósforo sérico após o tratamento } \\
\text { em nenhum dos grupos. } \\
\text { - O estudo não recomenda a ablação por micro- } \\
\text { ondas como tratamento de primeira linha em } \\
\text { pacientes com HPTS grave em hemodiálise de } \\
\text { manutenção. }\end{array}$ \\
\hline $\begin{array}{l}\text { Pharmacokinetics of a } \\
\text { New Oral Vitamin D } \\
\text { Receptor Activator (2- } \\
\text { Methylene-19-Nor- } \\
\text { (20S)-1a,25- } \\
\text { Dihydroxyvitamin D3) in } \\
\text { Patients with Chronic } \\
\text { Kidney Disease and } \\
\text { Secondary } \\
\text { Hyperparathyroidism on } \\
\text { Hemodialysis }\end{array}$ & $\begin{array}{c}\text { Estudo } \\
\text { observacional } \\
\text { analítico }\end{array}$ & 2017 & Pandey et al. & $\begin{array}{l}\text { 2-Metileno-19-nor- } \\
\text { (20S)-1 } \alpha \\
\text { 25-dihidroxivitamina } \\
\text { D3 (DP001) }\end{array}$ & $\begin{array}{l}\text { - DP001 mostrou um grande volume de } \\
\text { distribuição, indicando que apenas uma pequena } \\
\text { fração da dose permaneceu no plasma e a droga foi } \\
\text { altamente distribuída no tecido. } \\
\text { - A avaliação farmacodinâmica na última semana } \\
\text { de estudo mostrou que o iPTH permanece } \\
\text { suprimido ao longo de um período de } 48 \text { horas } \\
\text { durante o tempo em que os níveis do medicamento } \\
\text { no sangue estão mudando. Além disso, os níveis da } \\
\text { droga no sangue não são preditivos da resposta } \\
\text { iPTH. } \\
\text { - Nenhum indivíduo atingiu ou ultrapassou o limite }\end{array}$ \\
\hline
\end{tabular}




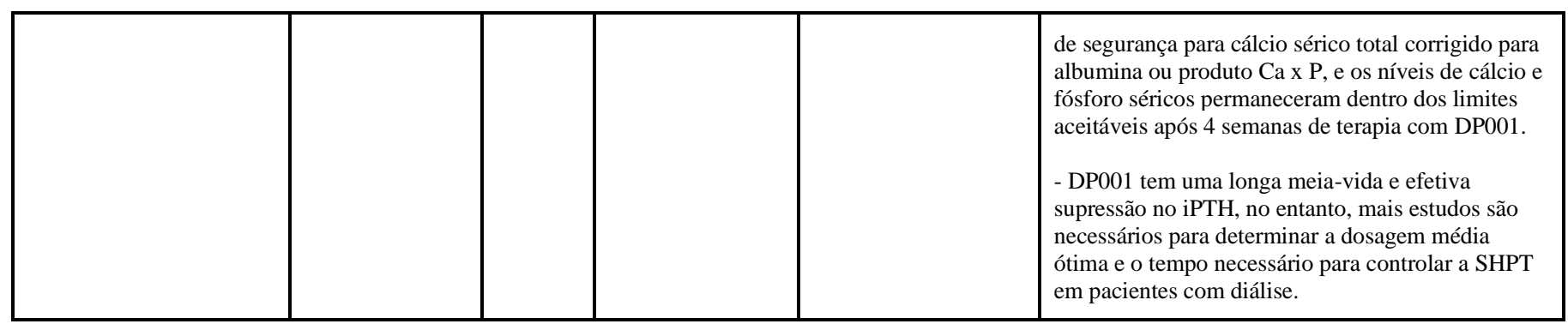

Fonte: Autores.

\section{Cinacalcet}

Em estudos realizados por Akizawa et al. (2016) e Susantitaphong et al. (2019) em pacientes em hemodiálise com HPTS, o cálcio-mimético foi capaz de reduzir efetivamente os níveis de iPTH e de cálcio sérico basal, além de estabilizar a calcificação vascular, reduzir a mortalidade e hospitalização por causa cardiovascular em pacientes com DRC em estágio 5D e, ainda, promover melhora nos marcadores de doença renal crônica e distúrbios ósseos minerais (DRC-DOM) e no fator de crescimento fibroblástico 23 (FGF-23) - este último, inclusive, em pacientes em hemodiálise crônica com níveis médios de PTH por volta de 1400 pg/mL -, apesar destes terem sofrido notório efeito rebote após descontinuação do fármaco, sugerindo que o tratamento a longo-prazo é necessário para o controle dessas desordens (Akizawa et al., 2016; Susantitaphong et al., 2019).

Para Akizawa et al. (2016), os benefícios aparentes do cinacalcet foram maiores em pacientes com iPTH basal $\geq 500$ pg/ml. Paralelamente, a meta-alvo do hormônio estabelecida por Susantitaphong et al. (2019) foi mais bem observada nos pacientes com níveis prévios de aproximadamente 800-1600 pg/mL. Porém, os estudos discordam quanto à ação na hiperplasia da paratireoide (prevenindo, assim, a não responsividade à terapia farmacológica), pois segundo Akizawa et al. (2016), o efeito redutor do volume da glândula é maior em pacientes com níveis séricos de iPTH mais elevados, enquanto que para Susantitaphong et al. (2019) estes achados foram significativos em pacientes com iPTH por volta de $800-1600 \mathrm{pg} / \mathrm{mL}$, implicando que níveis acima de 1600 pg/mL devem ser aconselhados à cirurgia.

Em conclusão, o fármaco pode promover efeitos salutares na DCR-DMO em pacientes em hemodiálise crônica com HPTS grave e compõe uma terapia inicial efetiva para reduzir o PTH prioritária à cirurgia, bem como uma alternativa terapêutica para aqueles que não se encaixam nos critérios cirúrgicos (Susantitaphong et al., 2019), entretanto, os efeitos adversos desencadeados pela droga e a sua associação a várias interações medicamentosas culminam na baixa adesão ao tratamento pelos pacientes e doses insuficientes (Fukagawa et al., 2017; Akizawa; Shimazaki; Fukagawa, 2018).

\section{Paratireoidectomia}

Apesar da PTX poder reduzir de maneira eficaz os níveis de iPTH na maioria dos pacientes, a melhora no controle bioquímico no pós-cirúrgico pode não ser perfeita (Susantitaphong et al., 2019). Segundo Akizawa, Shimazaki, e Fukagawa (2018), um alto nível de iPTH (> 500 pg/mL) consiste em uma das indicações para PTX.

Cruzado et al. (2016) demonstrou que a PTX subtotal é superior ao cinacalcet para corrigir hipercalcemia em pacientes transplantados renais com HPT persistente: todos os pacientes submetidos à cirurgia e $\leq 70 \%$ com cinacalcet atingiram normocalcemia; ainda, a redução do PTH foi maior no grupo da PTX, e apenas este foi associado com melhora significativa da densidade óssea mineral no colo femoral.

A cirurgia subtotal foi associada com melhora na densidade mineral e regulação dos biomarcadores de volume ósseo (atribuída possivelmente à normalização do PTH e/ou a suplementação oral de cálcio e vitamina D dada após a cirurgia para prevenir hipocalcemia associada à síndrome do osso faminto), porém não foi associada com regressão da calcificação vascular, 
embora a correção quase total do cálcio sérico e PTH tenha sido alcançada - e ambos os tratamentos (PTX subtotal e cinacalcet) apresentaram resultados similares na calcificação vascular, associados com sua não progressão -, sugerindo que essa patologia não é reversível, ao menos a curto-prazo, o que reforça a importância de prevenir desordens minerais ósseas nos estágios iniciais da DRC (Cruzado et al., 2016).

Redução da taxa de filtração glomerular foi observada em ambos os grupos, embora mais acentuada com o cinacalcet, fato o qual não foi possível justificativa, e os efeitos adversos gastrointestinais (GI ) observados nos pacientes tratados com o fármaco podem ser associados à hipovolemia e disfunção renal (Cruzado et al., 2016).

A hospitalização precoce foi maior após a PTX, sendo a principal complicação a hipocalcemia, relacionada à intolerância oral causada por altas doses de cálcio e disfonia transiente causada por traumatismo cirúrgico leve do nervo laríngeo recorrente (Cruzado et al., 2016).

De acordo com estudo desenvolvido por Zhang et al. (2016), foi demonstrada forte e independente associação entre baixos níveis séricos de leptina (hormônio peptídico secretado pelos adipócitos e eliminado pelos rins) e baixo IMC, anemia (hiperleptinemia mostrou-se fator estimulante para eritropoiese, refletindo melhor resposta da eritropoietina em pacientes em diálise de longo-prazo) e níveis reduzidos de albumina em pacientes DCR.

Devido a normalização dos níveis de PTH pela PTX, aumentando os níveis circulantes da adipocina (pois o PTH elevado reduz a produção ao inibir a fosforilação do Akt nos adipócitos), foi possível melhorar a anemia e mal nutrição em pacientes com HPTS grave (Zhang et al., 2016).

Além disso, a leptina pode ser promissora para gerar desfechos favoráveis em pacientes DCR com dislipidemia e estes elementos contribuem para que seja utilizada como um dos biomarcadores para essas enfermidades, abrindo novas possibilidades de prevenção e intervenção terapêutica em pacientes DCR (Zhang et al., 2016).

\section{Etelcalcetide}

Em estudo desenvolvido por Fukagawa et al. (2017), a administração de etelcalcetide três vezes por semana durante 12 semanas reduziu o iPTH sérico com significância estatística em comparação com o placebo e, uma vez que a apresentação intravenosa pode ser facilmente administrada por retorno do sangue após a diálise, possibilita uma redução na carga de comprimidos e fornece uma nova alternativa para maior adesão dos pacientes ao tratamento de HPTS.

Resultados semelhantes foram obtidas por Bushinsky et al. (2020), com redução sustentada nos níveis séricos de PTH ( $68 \%$ dos pacientes alcançaram redução maior do que $30 \%$ e 56\%, PTH igual a 300 pg $/ \mathrm{mL}$ ), cálcio e fósforo. Além destes, pacientes randomizados com etelcalcetide foram mais favoráveis a experienciar reduções substanciais do FGF-23 (Block et al., 2017).

Com relação ao perfil de segurança, Block et al. (2017, 2019), Fukagawa et al. (2017) e Bushinsky et al. (2020) demonstraram, a partir de ensaios clínicos com pacientes com HPTS em hemodiálise, que o etelcalcetide é bem tolerado e semelhante ao cinacalcet, sendo as reações adversas majoritariamente ligadas à diminuição do cálcio sérico (QT prolongado, parestesia, espasmos musculares e mialgia) ou relacionados a sintomas GI (diarreia, náusea e vômito), sendo pouco frequente a necessidade de descontinuação da droga.

A maioria dos casos de hipocalcemia foram de severidade média ou moderada, com taxas semelhantes aos pacientes tratados com cinacalcet; porém, ressalta-se que os cálcio-miméticos não devem ser administrados concomitantemente por conferirem risco aumentado de hipocalcemia pela ação sinérgica no receptor, além da importância do monitoramento frequente do cálcio sérico e de outros marcadores do metabolismo ósseo (Block et al., 2019).

Bushinsky et al. (2020) sugerem que o tratamento de longo prazo com etelcalcetide não aumenta o risco de hipocalcemia ou provoca risco mínimo. Hipercalemia foi reportada com maior frequência em Block et al. (2017), porém não 
foram evidenciados fatores de risco consistentes ou eventos associados. Não houve aumento na incidência de efeitos adversos potencialmente associados ao prolongamento de QT, nem evidência de um efeito direto na repolarização cardíaca mas, apesar disso, é preciso cuidado quanto ao uso do fármaco em conjunto com outras drogas que possam prolongar o intervalo QT (Block et al., 2017, 2019).

As taxas de fratura com o uso de etelcalcetide foram quase metade das observadas no grupo placebo (apesar da ocorrência de níveis de PTH < 100pg/mL); entretanto, é possível que a redução mediada a nível de cálcio possa desencadear instabilidade hemodinâmica e que a hipocalcemia rápida ou prolongada pode potencialmente reduzir a contratilidade do miocárdio, principalmente em pacientes com função cardíaca deficiente (Block et al., 2019).

Há risco baixo a nulo de interações com outras drogas, porém interações farmacodinâmicas podem ocorrer com aqueles que reduzem o cálcio sérico (Block et al., 2019) e os ensaios demonstraram a importância da estratégia de ajuste das doses em relação a uma fixa, de acordo com a exposição a efeitos adversos graves (Block et al., 2017; Bushinski et al., 2020).

\section{Evocalcet}

Yokoyama et al. (2019), ao examinar a eficácia e segurança a longo prazo do novo cálcio-mimético em pacientes japoneses em hemodiálise com HPTS, observaram contínua redução do iPTH até atingir a meta alvo (entre 60-240 pg/mL) com a administração contínua de evocalcet. A maioria deles, entretanto, utilizou previamente o cinacalcet, levando a assumir que os valores paramétricos basais tenham sido influenciados pelo tratamento prévio; no entanto, mesmo nestes pacientes, a concentração de iPTH diminuiu ainda mais após a troca para o evocalcet (Yokoyama et al., 2019).

Embora os níveis de iPTH, cálcio e fósforo tenham aumentado temporariamente após a troca, tais flutuações podem ser atribuídas à dosagem pré-terapêutica de cinacalcet, ou ainda, à dose inicialmente administrada relativamente baixa de evocalcet (Yokoyama et al., 2019). Outros estudos apresentaram resultados semelhantes de correção dos níveis de iPTH e cálcio de forma proporcional à dose, o fármaco exercendo efeito terapêutico superior em relação ao placebo a partir de $1 \mathrm{mg}, \mathrm{e}$ em doses mais baixas do que o cinacalcet, melhorando sua adesão (Akizawa et al., 2018; Akizawa; Shimazaki; Fukagawa, 2018; Shigematsu et al., 2019). O tratamento com 1 e 4 mg no período de doses múltiplas mostrou que é improvável que a hemodiálise influencie as taxas de depuração (Shigematsu et al., 2019).

O novo fármaco foi, de maneira geral, bem tolerado nas doses administradas, embora níveis diminuídos de cálcio no sangue tenham sido relatados no estudo de Shigematsu et al. (2019) e, além disso, não houve diferenças no perfil de segurança entre pacientes pré-tratados com cinacalcet e os que não o foram (Akizawa et al., 2018; Shigematsu et al., 2019; Yokoyama et al., 2019).

Os efeitos adversos mais significativos foram considerados leves, com os GI pouco relacionados ou ausentes (contribuindo para sua superioridade em relação ao cinacalcet), e os graves incluíram insuficiência cardíaca congestiva e cardiomiopatia, porém, com ínfima frequência, e apresentaram melhora ou foram resolvidos com ou sem tratamento (Akizawa et al., 2018; Shigematsu et al., 2019; Yokoyama et al., 2019).

A respeito de alterações eletrocardiográficas, Shigematsu et al. (2019) correlacionaram associação entre o prolongamento do QT corrigido de Fridericia e a administração de evocalcet, uma vez que os cálcio-miméticos induzem a diminuição do cálcio no sangue. Não ocorreram eventos significativos atribuídos ao aumento da dose de evocalcet, permitindo sua administração a longo-prazo e melhorando a eficácia da droga, além de que o tratamento prévio com cinacalcet não ofereceu preocupações quanto à segurança dos pacientes japoneses tratados (Yokoyama et al., 2019) e, dessa forma, o perfil de segurança do evocalcet oferece uma alternativa potencial aos cálcio-miméticos existentes para tratamento de HPTS (Shigematsu et al., 2019). 
Em outro estudo com pacientes japoneses realizando diálise peritoneal, a administração com ajuste de dose de evocalcet uma vez por dia durante 52 semanas possibilitou o alcance da meta de iPTH entre 60-240 pg/mL (Tsuruya et al., 2019). Adicionalmente, níveis corrigidos de cálcio e fósforo séricos permaneceram constantes até a última semana e não foram observadas novas preocupações quanto à segurança (incluindo efeitos adversos GI), nem reportadas reações adversas severas à droga, caracterizando o fármaco como uma opção mais tolerada para pacientes com HPTS (Tsuruya et al., 2019).

\section{Outros}

Pacientes com DRC são propensos a desenvolver deficiência de calcidiol [25-hidroxivitamina D (25 (OH) D)], a qual está associada a um aumento no PTH em indivíduos saudáveis e comprometimento da função muscular e óssea e, para tanto, as diretrizes clínicas recomendam reposição com vitamina D nutricional para atingir uma concentração plasmática ideal (Westerberg et al., 2018).

Em ensaio clínico conduzido com pacientes nos estágios 3 e 4 da DRC com HPTS, Westerberg et al. (2018) mostraram que o colecalciferol em altas doses (8.000 UI/dia) alivia o desenvolvimento da patologia secundária à DRC ao atingir a suficiência completa de $25(\mathrm{OH}) \mathrm{D}$ e induzir aumento substancial do 1,25 (OH) D (calcitriol), evitando assim a tendência à hipocalcemia que de outra forma se desenvolve.

Paralelamente, não é causada hipercalcemia ou perda acelerada da taxa de filtração glomerular, nem induzido aumento de fosfato ou FGF-23, mas também não há melhora na fadiga ou força de preensão manual (Westerberg et al., 2018). Em adição, Zheng et al. (2016) revelaram que acredita-se que a saturação de $25(\mathrm{OH}) \mathrm{D} 3 \mathrm{com}$ colecalciferol em alta dose atua conjuntamente com o cinacalcet em dose baixa e análogos ativos de vitamina D para potencializar a inibição da secreção de PTH e melhorar a densidade mineral óssea do colo do fêmur em pacientes em diálise.

O calcitriol é utilizado para suprimir o PTH em pacientes com HPTS. Contudo, ele apresenta como efeito adverso o aumento da concentração sérica de cálcio e de fosfato e, nesse sentido, foram desenvolvidos análogos do calcitriol como o paricalcitol o qual, embora possua ação menos potente, também é potencialmente menos calcêmico do que o calcitriol (Pandey et al., 2017).

Em estudos clínicos prospectivos conduzidos para avaliar a farmacocinética, eficácia e segurança do paricalcitol oral em crianças de 10 a 16 anos com DRC nos estágios 3-5, observou-se que o fármaco é eficaz na redução dos níveis de iPTH sem normalmente elevar cálcio e fósforo e pode ter um potencial ainda maior para controlar a renovação óssea enquanto minimiza o risco de efeitos adversos no crescimento ósseo linear, além de ter sido bem tolerado por longos períodos de teste e a administração parece ser semelhante à observada em adultos, sustentando a dose inicial de $1 \mu \mathrm{g}$ em estudos futuros e na prática clínica (Webb et al., 2017).

Olaizola et al. (2016) avaliaram a eficácia e segurança do paricalcitol endovenoso administrado durante seis meses em pacientes com HPTS em diálise, virgens de tratamento com metabólitos de vitamina $\mathrm{D}$ ou que não responderam às terapias atuais e, apesar das limitações, concluíram que a droga é eficaz na diminuição do iPTH, além de ter havido nível sérico médio de cálcio aceitável ao final do estudo e nenhuma manifestação clínica de hipercalcemia ou aumento significativo dos níveis médios de fósforo, sendo o efeito adverso mais relatado a hiperfosfatemia. Portanto, consideram adequada a introdução de derivados da vitamina D (particularmente seus análogos como o paricalcitol) tendo em conta a sua eficácia e tolerância, principalmente em níveis de PTH entre 150 e 800 pg/mL (Olaizola et al., 2016).

A farmacocinética e os benefícios do DP001, novo ativador do receptor de vitamina D com alta seletividade para o osso e a glândula paratireoide, cuja ação é suprimir a produção de PTH, foram demonstrados por Pandey et al. (2017), e observou-se que devido a concentração máxima (Cmáx) e a curva de relação plasmática e tempo (AUC) serem expressivamente mais baixas e o volume de distribuição, maior do que os do calcitriol oral e do paricalcitol, grande parte do 
fármaco é rapidamente distribuída para alvos periféricos onde exerce ação, e, além disso, o tempo de meia-vida do DP001 nos pacientes em hemodiálise apresentou-se notavelmente maior. O estudo ressalta, ainda, a alta potência do DP001 como análogo da vitamina D (administração de uma dose de 550 ng oral, 3x por semana levou à supressão do iPTH por 4 semanas e metade dos pacientes tratados responderam com pelo menos 30\% da redução) e foi ilustrado que a supressão efetiva do iPTH de forma prolongada ocorre quando o DP0001 é fornecido a cada 2-3 dias, adicional à capacidade de supressão sem provocar efeitos de hipercalcemia e hiperfosfatemia, porém mais estudos são necessários para determinar a dosagem média ótima e o tempo necessário para controle (Pandey et al., 2017).

A ablação por micro-ondas (AMO), que se tornou um meio minimamente invasivo cada vez mais popular de tratamento de tumores, também tem sido utilizada no HPTS grave, e sua eficácia e segurança foram testadas por Diao et al. (2017) em pacientes em hemodiálise de manutenção. Todavia, os resultados sobre os parâmetros bioquímicos e a porcentagem de pacientes com hipocalcemia (50\% da amostra) não foram satisfatórios, indicando baixa eficácia, sendo as possíveis explicações dadas: a possibilidade de ter havido células paratireoides residuais após a ablação, que com o metabolismo desordenado do cálcio e fósforo em curso em pacientes dialisados tenderiam a proliferar e o HPTS reapareceria; os pesquisadores não conseguiram fazer a ablação de todos os nódulos em todos os pacientes e talvez houvesse tecido ectópico ativo não detectável por ultrassom; e a eficácia é altamente dependente da técnica do operador (Diao et al., 2017). Dessa forma, apesar das limitações, não foi recomendada AMO como tratamento de primeira linha em pacientes com HPTS grave em hemodiálise de manutenção (Diao et al., 2017).

\section{Conclusão}

A partir dessa análise foi possível atestar a variedade de novos estudos na busca de um tratamento medicamentoso mais promissor e de mais amplo uso no HPTS em detrimento da PTX. Novos cálcio-miméticos como etelcalcetide e evocalcet demonstraram experimentalmente ser uma alternativa ao tratamento com cinacalcet, uma vez que possuem níveis similares de eficácia e segurança aos do medicamento clássico, mas com vantagens em relação ao uso, e por consequência, na adesão dos pacientes ao tratamento. Outra linha de pesquisa promissora refere-se ao uso de análogos e precursores de vitamina $\mathrm{D}$, que apresentam bons resultados na diminuição do iPTH. Alternativas ao calcitriol surgem na intenção de suplantar os efeitos adversos desse medicamento, e, dessa forma, o colecalciferol e o paricalcitol mostraram resultados satisfatórios em diminuir o iPTH sérico e controlar a renovação óssea sem apresentar os efeitos adversos de hipercalcemia, quando utilizados nas doses corretas. Além disso, o DP001 demonstra-se um análogo altamente potente da vitamina D, com capacidade de suprimir efetivamente o iPTH de forma prolongada sem efeitos de hipercalcemia e hiperfosfatemia. Entretanto, apesar desses avanços, todas as pesquisas analisadas possuíram problemas como pequena amostra, limitação na avaliação de todas as variáveis envolvidas no HPTS e as metodologias utilizadas, nas quais não foram exercidas comparações diretas dos recursos farmacológicos em questão com um espectro mais amplo de opções terapêuticas, como a própria PTX. Dessa forma, mesmo as terapias analisadas sendo potencialmente promissoras, mais estudos devem ser desenvolvidos, com a finalidade de definir recursos farmacológicos e técnicos capazes de se sobreporem aos tratamentos adotados atualmente.

Este artigo possui limitações, como o levantamento de literatura realizado em uma única base de dados e a quantidade relativamente pequena de estudos encontrados acerca de cada tipo de tratamento para o HPTS, o que acarretou um número reduzido de referências utilizadas (e a repetição das mesmas) e um risco potencialmente maior de geração de viés. Portanto, sugere-se que pesquisas futuras sejam efetuadas abrangendo uma maior variedade de bancos de dados, de maneira a haver uma melhor comparação entre as terapias já utilizadas ou em desenvolvimento, e para que o repasse de informações seja de confiabilidade mais adequada. 


\section{Referências}

Akizawa, T., Kurita, N., Mizobuchi, M., Fukagawa, M., Onishi, Y., Yamaguchi, T., ... \& Fukuhara, S. (2016). PTH-dependence of the effectiveness of cinacalcet in hemodialysis patients with secondary hyperparathyroidism. Scientific reports, 6(1), 1-11.

Akizawa, T., Shimazaki, R., Shiramoto, M., \& Fukagawa, M. (2018). Pharmacokinetics, pharmacodynamics, and safety of the novel calcimimetic agent evocalcet in healthy Japanese subjects: first-in-human phase I study. Clinical drug investigation, 38(10), 945-954.

Akizawa, T., Shimazaki, R., Fukagawa, M., \& Evocalcet Study Group. (2018). Phase 2b study of evocalcet (KHK7580), a novel calcimimetic, in Japanese patients with secondary hyperparathyroidism undergoing hemodialysis: A randomized, double-blind, placebo-controlled, dose-finding study. PloS one, 13(10), e0204896.

Bilezikian, J. P., Bandeira, L., Khan, A., \& Cusano, N. E. (2018). Hyperparathyroidism. The Lancet, 391(10116), 168-178.

Block, G. A., Bushinsky, D. A., Cunningham, J., Drueke, T. B., Ketteler, M., Kewalramani, R., ... \& Chertow, G. M. (2017). Effect of etelcalcetide vs placebo on serum parathyroid hormone in patients receiving hemodialysis with secondary hyperparathyroidism: two randomized clinical trials. Jama, 317(2), 146-155.

Block, G. A., Chertow, G. M., Sullivan, J. T., Deng, H., Mather, O., Tomlin, H., \& Serenko, M. (2019). An integrated analysis of safety and tolerability of etelcalcetide in patients receiving hemodialysis with secondary hyperparathyroidism. PloS one, 14(3), e0213774.

Bringhurst, F. R. (1998). Hormones and disorders of mineral metabolism. Williams Textbook of Endocrinology., $1155-1200$.

Bushinsky, D. A., Chertow, G. M., Cheng, S., Deng, H., Kopyt, N., Martin, K. J., ... \& Block, G. A. (2020). One-year safety and efficacy of intravenous etelcalcetide in patients on hemodialysis with secondary hyperparathyroidism. Nephrology Dialysis Transplantation, 35(10), 1769-1778.

Cruzado, J. M., Moreno, P., Torregrosa, J. V., Taco, O., Mast, R., Gómez-Vaquero, C., ... \& Grinyó, J. M. (2016). A randomized study comparing parathyroidectomy with cinacalcet for treating hypercalcemia in kidney allograft recipients with hyperparathyroidism. Journal of the American Society of Nephrology, 27(8), 2487-2494.

Custódio, M. R., Canziani, M. E. F., Moysés, R. M. A., Barreto, F. C., Neves, C. L., Oliveira, R. B. D., ... \& Carvalho, A. B. D. (2013). Protocolo clínico e diretrizes terapêuticas para o tratamento do hiperparatireoidismo secundário em pacientes com doença renal crônica. Brazilian Journal of Nephrology, 35, 308322.

Diao, Z., Wang, L., Li, D., \& Liu, W. (2017). Efficacy of microwave ablation for severe secondary hyperparathyroidism in subjects undergoing hemodialysis. Renal failure, 39(1), 140-145.

Fukagawa, M., Yokoyama, K., Shigematsu, T., Akiba, T., Fujii, A., Kuramoto, T., ... \& ONO-5163 Study Group. (2017). A phase 3, multicentre, randomized, double-blind, placebo-controlled, parallel-group study to evaluate the efficacy and safety of etelcalcetide (ONO-5163/AMG 416), a novel intravenous calcimimetic, for secondary hyperparathyroidism in Japanese haemodialysis patients. Nephrology Dialysis Transplantation, 32(10), 1723-1730.

Golan, David E. et al. (2014) Princípios de farmacologia: a base fisiopatológica da farmacoterapia. 3. ed. Rio de Janeiro: Guanabara Koogan.

Guyton, A.C.; Hall, J.E. (2011) Tratado de Fisiologia Médica. 12. ed. Rio de Janeiro: Elsevier.

Neves, P. D. M. D. M., Sesso, R. D. C. C., Thomé, F. S., Lugon, J. R., \& Nasicmento, M. M. (2020). Brazilian Dialysis Census: analysis of data from the 2009-2018 decade. Brazilian Journal of Nephrology, 42, 191-200.

Olaizola, I., Caorsi, H., Fajardo, L., Ferreiro, A., Campistrus, N., Dolinsky, D., ... \& Ambrosoni, P. (2016). Effectiveness and safety of a 6-month treatment with paricalcitol in patients on hemodialysis with secondary hyperparathyroidism. Brazilian Journal of Nephrology, 38, 302-312.

Pandey, R., Zella, J. B., Zhu, J. G., Plum, L. A., Clagett-Dame, M., Blaser, W. J., ... \& Coyne, D. W. (2017). Pharmacokinetics of a New Oral Vitamin D Receptor Activator (2-Methylene-19-Nor-(20S)-1 $\alpha$, 25-Dihydroxyvitamin D 3) in Patients with Chronic Kidney Disease and Secondary Hyperparathyroidism on Hemodialysis. Drugs in $R \& D, 17(4), 597-605$.

Porto, R. A., Truite, M. R., Bucharles, S. E., \& Hauser, A. B. (2016). Hiperparatireoidismo secundário: uma complicação da Doença Renal Crônica. Rev. Bras. Anal. Clin, 48(3), 182-188.

SBEM (Sociedade Brasileira de Endocrinologia e Metabologia). (2006) Hiperparatireoidismo Primário, Projeto Diretrizes.

Shigematsu, T., Shimazaki, R., Fukagawa, M., Akizawa, T., \& Evocalcet Study Group. (2019). Pharmacodynamics of evocalcet for secondary hyperparathyroidism in Japanese hemodialysis patients. Clinical and experimental nephrology, 23(2), 258-267.

Susantitaphong, P., Vadcharavivad, S., Susomboon, T., Singhan, W., Dumrongpisutikul, N., Jakchairoongruang, K., ... \& Praditpornsilpa, K. (2019). The effectiveness of cinacalcet: a randomized, open label study in chronic hemodialysis patients with severe secondary hyperparathyroidism. Renal failure, $41(1)$, 326-333.

Tsuruya, K., Shimazaki, R., Fukagawa, M., \& Akizawa, T. (2019). Efficacy and safety of evocalcet in Japanese peritoneal dialy sis patients. Clinical and experimental nephrology, 23(6), 739-748.

Webb, N. J., Lerner, G., Warady, B. A., Dell, K. M., Greenbaum, L. A., Ariceta, G., ... \& Dufek, M. B. (2017). Efficacy and safety of paricalcitol in children with stages 3 to 5 chronic kidney disease. Pediatric Nephrology, 32(7), 1221-1232.

Westerberg, P. A., Sterner, G., Ljunggren, Ö., Isaksson, E., Elvarson, F., Dezfoolian, H., \& Linde, T. (2018). High doses of cholecalciferol alleviate the progression of hyperparathyroidism in patients with CKD Stages 3-4: results of a 12-week double-blind, randomized, controlled study. Nephrology Dialysis Transplantation, 33(3), 466-471. 
Research, Society and Development, v. 11, n. 1, e11011123608, 2022

(CC BY 4.0) | ISSN 2525-3409 | DOI: http://dx.doi.org/10.33448/rsd-v11i1.23608

Xavier, Cláudia M. (2020) Tratamento cirúrgico do hiperparatireoidismo primário.

Yokoyama, K., Shimazaki, R., Fukagawa, M., \& Akizawa, T. (2019). Long-term efficacy and safety of evocalcet in Japanese patients with secondary hyperparathyroidism receiving hemodialysis. Scientific reports, 9(1), 1-11.

Jiang, Y., Zhang, J., Yuan, Y., Zha, X., Xing, C., Shen, C., ... \& Wang, N. (2016). Association of increased serum leptin with ameliorated anemia and malnutrition in stage 5 chronic kidney disease patients after parathyroidectomy. Scientific reports, 6(1), 1-13.

Zheng, J. Q., Hou, Y. C., Zheng, C. M., Lu, C. L., Liu, W. C., Wu, C. C., ... \& Lu, K. C. (2016). Cholecalciferol additively reduces serum parathyroid hormone and increases vitamin D and cathelicidin levels in paricalcitol-treated secondary hyperparathyroid hemodialysis patients. Nutrients, 8(11), 708. 